\title{
Modulating the Antioxidant Activity of Thin Layer-by-Layer Films with Polyphenols
}

ISSN: 2637-8078

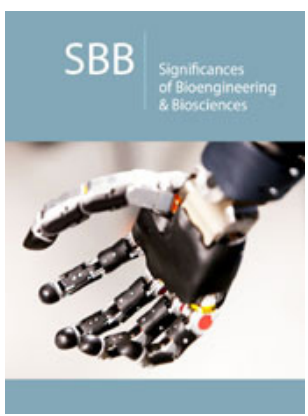

*Corresponding author: Vincent Ball, Institute National de la Santé et de la Recherche Médicale, France

Submission: 侮-19y 21, 2019

Published: 海 May 30, 2019

Volume 3 - Issue 2

How to cite this article: Vincent $B$. Modulating the Antioxidant Activity of Thin Layer-by-Layer Films with Polyphenols.Significances Bioeng Biosci.3(2). SBB.000560.2019.

DOI: 10.31031/SBB.2019.03.000560

Copyright@ Vincent Ball, This article is distributed under the terms of the Creative Commons Attribution 4.0 International License, which permits unrestricted use and redistribution provided that the original author and source are credited.

\author{
Vincent Ball* \\ Institute National de la Santé et de la Recherche Médicale, France
}

\begin{abstract}
Layer-by-layer deposition of a polycation and of a cheap polyphenol, tannic acid, allows to produce thin coatings having an antioxidant activity proportional to the amount of deposited polyphenol. This means that the used probe, 2,2-diphenyl-1-picrylhydrazyl (DPPH), is able to reach all tannic acid molecules present in the film whatever their location. However, when the tannic acid containing film is capped with a few nanometers thick capping layer made of poly (allylamine hydrochloride) and poly (sodium 4-styrene sulfonate), the DPPH has no access anymore to the embedded tannic acid. On this basis, an application is proposed for the production of a packaging film containing tannic acid as a probe able to sense if the packaging has undergone some mechanical damage.
\end{abstract}

Keywords: Tannic acid; Layer-by-layer deposition; Antioxidant activity

\section{Introduction}

Plants and fruits contain many antioxidant molecules among them polyphenols [1]. Such natural antioxidants can find applications in food science [2] and in particular for food packaging when the molecules of interest are integrated in a film or a membrane. Many polyphenols, among them tannic acid (Figure 1) deposit spontaneously at solid liquid interfaces in a versatile manner. The deposition of TA and other polyphenols occurring spontaneously on glasses from wine or from tea has been exploited recently in materials science [3,4]. Polyphenols contain redox active groups which can also act as coordination centers for metal cations like $\mathrm{Fe}^{3+}$. This chemical modality allows the deposition from polyphenol-metal cation mixtures to yield conformal coatings [5] or to obtain films by alternating adsorption steps of the polyphenol and metal cations according to a Layer-by-Layer (LBL) deposition method [6]. The same deposition strategy can be used to deposit polyphenol containing films by alternating their adsorption with that of a polymer [7], a polyelectrolyte [8] or proteins [9]. In these cases, the interactions responsible for the cohesion of the films are due to hydrogen bonding or to electrostatic interactions (polyphenols containing also weakly acidic groups able to become anionic at high enough $\mathrm{pH}$ ). The deposition of films made from proteins and polyphenols is due to the interplay of many kinds of interactions among them some specific interactions with amino acids like L-proline [10]. Films containing TA and other polyphenols display some antioxidant activity [11]. The advantage of the LBL deposition method of a material with respect to direct functionalization by contact with a polyphenol containing solution, leading mostly to a deposited monolayer, is an increased stability and durability of the coating. Herein, it will be shown, that LBL films made from a polycation and TA are characterized by an amount of TA which is proportional to the number of deposition steps.

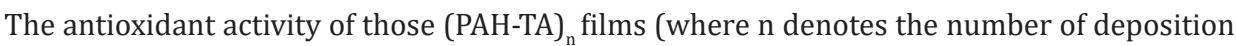
cycles of the polycation and the polyphenol) scales also proportionally with the number of the deposition steps, as in other studies [11]. This means that all the polyphenol molecules in the film are accessible to the used probe, 2,2-diphenyl-1-picrylhydrazyl (Figure 1). The antioxidant properties of the films can be suppressed by depositing two capping bilayers made from poly (allylamine hydrochloride) (PAH) and poly (sodium 4-styrene sulfonate) (PSS). This new finding will be discussed in terms of possible applications in food packaging.

\section{Material and Methods}

TA (ref. 403040, Mw=1701g. $\mathrm{mol}^{-1}$ ), PAH (ref. 283218, Mw=15000g.mol ${ }^{-1}$ obtained by means of gel permeation chromatography as given by the furnisher) and PSS (ref. 24,305 
1, Mw=70000g.mol-1 obtained by means of gel permeation chromatography as given by the furnisher) were purchased from Sigma Aldrich and used without further purification. All solutions were prepared before use in $50 \mathrm{mM}$ sodium acetate buffer. The buffer was made from double distilled and deionized water (Millipore D8 system, $\rho=18.2 \mathrm{M} \Omega . \mathrm{cm}$ ). TA, PAH and PSS were dissolved in the sodium acetate buffer at a concentration of $1 \mathrm{mg} \cdot \mathrm{mL}^{-1}$. DPPH (ref. D9132, Sigma-Aldrich) was dissolved at $10^{-4} \mathrm{M}$ in absolute ethanol and stored at $4{ }^{\circ} \mathrm{C}$ in the absence of light to ensure its stability. The chemical structure of TA and DDPH, as well as the reaction of DDPH with an oxidant are shown in Figure 1. The adsorption substrates were $4 \mathrm{~cm} \times 1 \mathrm{~cm}$ quartz slides (Thuet, Blodelsheim, France) cleaned with ethanol, dried under a stream of filtered air and subjected to 5 min of plasma cleaning in an air plasma (PDC-32G-2, Harrick Scientific, USA). The LBL deposition was performed as described in ref. [12]. Briefly: the cleaned quartz slide was dipped during $5 \mathrm{~min}$ in the PAH solution, in sodium acetate buffer, in the TA solution and again in sodium acetate buffer. The contact with buffer allowed to remove weakly bound molecules from the surface. The successive deposition of a PAH and TA led to the deposition of one-layer pair. This deposition step was repeated $\mathrm{n}$ times. The as obtained (PAH$\mathrm{TA})_{\mathrm{n}}$ films were then rinsed with distilled water and dried under a stream of filtered air, in order to avoid crystallization of salt from the buffer.

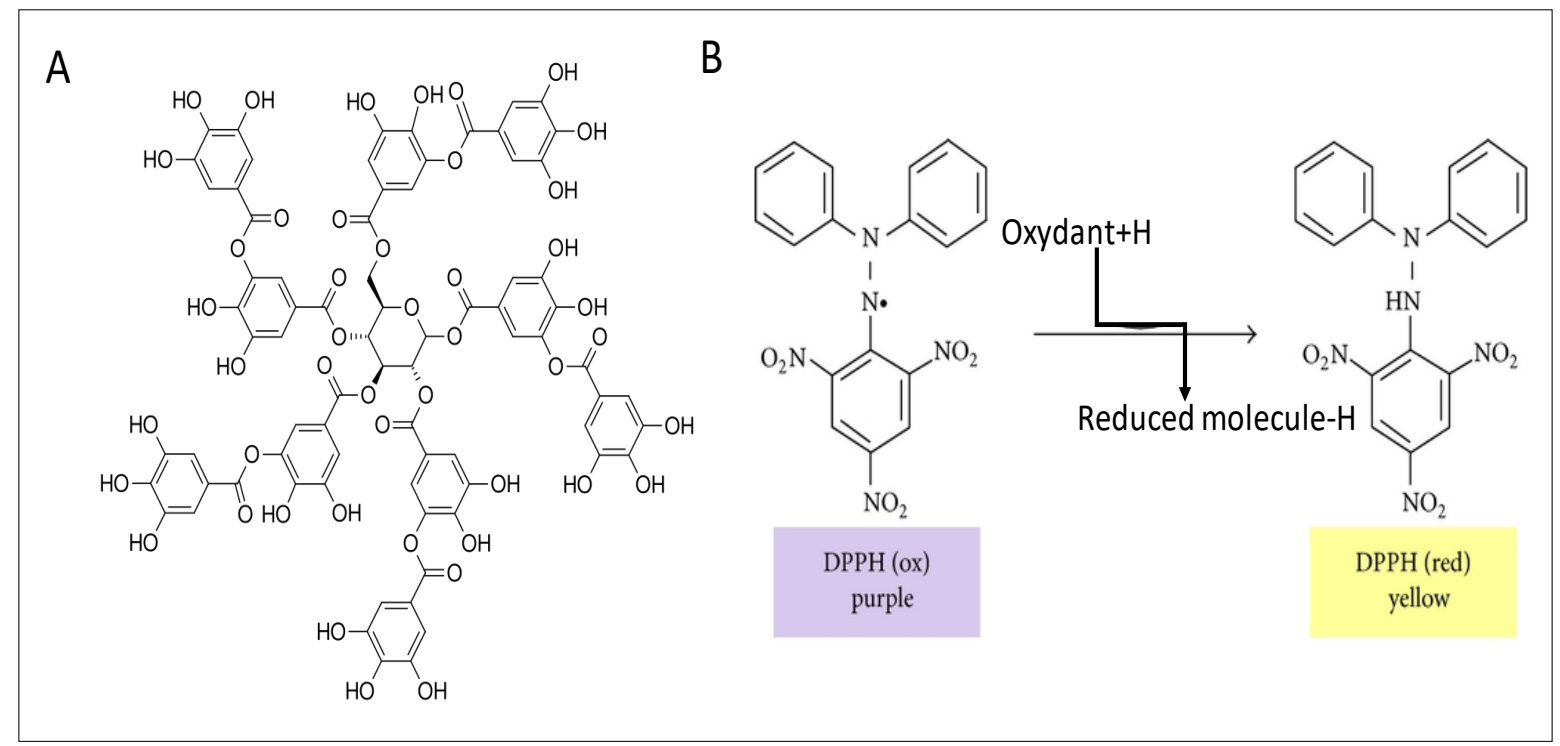

Figure 1: Chemical structure of TA (A) and of 2,2-diphenyl-1-picrylhydrazyl (DPPH) as well as the reaction undergone in the presence of an oxidant with the concomitant color change.

The absorbance of the quartz slide was then measured between 250 and $700 \mathrm{~nm}$ using a double beam spectrophotometer (UV$\mathrm{mc}^{2}$, Safas, Monaco) against a cleaned quartz slide taken as the reference. The films were then immersed in a $10^{-4} \mathrm{M}$ DPPH solution dissolved in absolute ethanol which constitutes time $t=0$ of the DPPH quenching kinetics. To follow that kinetics as a function of the number of PAH-TA layer pairs, the absorbance of the solution with the immersed quartz slide+film was measured every $15 \mathrm{~s}$ during $15 \mathrm{~min}$ at $\lambda=517 \mathrm{~nm}$ with the $\mathrm{mc}^{2}$ spectrophotometer. In these kinetic experiments, the reference cuvette contained the ethanolic DPPH solution and an uncoated quartz slide. Hence, the spontaneous quenching of DDPH by oxygen is taken into account. The absorbance will appear as negative values since the films induce a faster quenching than dissolved oxygen. To characterize the film deposition, water contact angles were measured with $5 \mu \mathrm{L}$ water droplets using the sessile drop method with an attention theta goniometer (Biolin Scientific, Sweden). The obtained contact angle values correspond to the average of 5 regularly spaced locations along the quartz slide and the error bar corresponds to one standard deviation. The water contact angle of LBL films characterizes their surface composition and a last layer effect is often observed for relatively stratified films [13].

\section{Result and Discussion}

The deposition of (PAH-TA) films (5min per adsorption and rinsing step as in ref. [12]) was characterized by means of UV-vis spectroscopy (Figure 2) and by contact angle goniometry (Figure 3 ). The film growth is of linear type, meaning that the absorbance increases at $\lambda=300 \mathrm{~nm}$, due to TA (PAH does not absorb light above $\lambda=200 \mathrm{~nm}$ ), scales in proportion to the number of deposited layer pairs (Figure 2). In addition, the interaction of the film with tiny water droplets reflects the nature of the last deposited layer (Figure 3 ). The films are more hydrophilic (static water contact angle close to $8^{\circ}$ ) when they are made from TA as the last deposited layer than from PAH as the last layer (contact angle close to $12^{\circ}$ ). The behavior found for (PAH-TA) films is typical of linearly growing LBL films $[6,11,13]$. The morphology of the (PAH-TA) $)_{n}$ films has also been characterized by means of Atomic Force Microscopy and some typical images acquired in the contact mode and in the dry state can be found in Figure 4 of ref. [12]. 


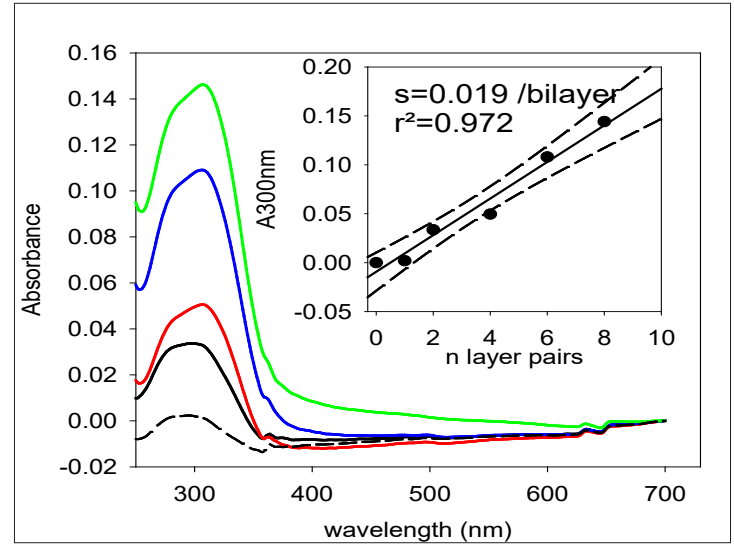

Figure 2: Absorbance spectra of (PAH-TA)n films with $\mathrm{n}=1\left({ }_{2}, \ldots\right), \mathrm{n}=2$ ), $n=4($ ), $\mathrm{n}=6($ and $n=8$ ) deposited layer pairs. The inset displays the absorbance increase at $\lambda=300 \mathrm{~nm}$ as a function of the number of deposited layer pairs. The full and dotted lines represent the linear regression to the data and the limit of the $95 \%$ confidence interval respectively. The (PAH-TA)n films were deposited on quartz slides and their spectra were measured against a plasma cleaned quartz slide.

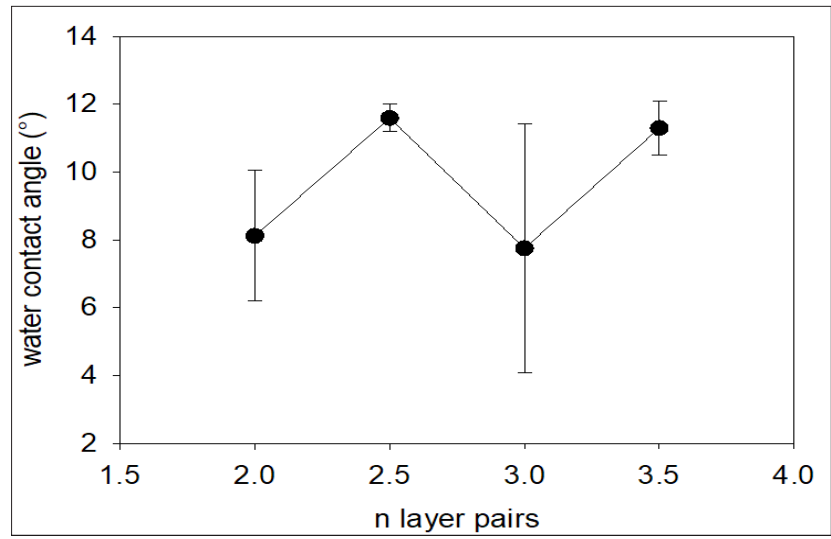

Figure 3: Evolution of the static water contact angles (obtained by the sessile drop method from $5 \mu \mathrm{L}$ drops) with the deposition of the (PAH-TA)n films. $n=2.5$ and 3.5 correspond to (PAH-TA)2-PAH and (PAH-TA)3-PAH films, hence to coatings ending with the deposition of the polycation whereas $n=2$ and 3 correspond to films with TA as the last deposition step. All points correspond to independently prepared films.

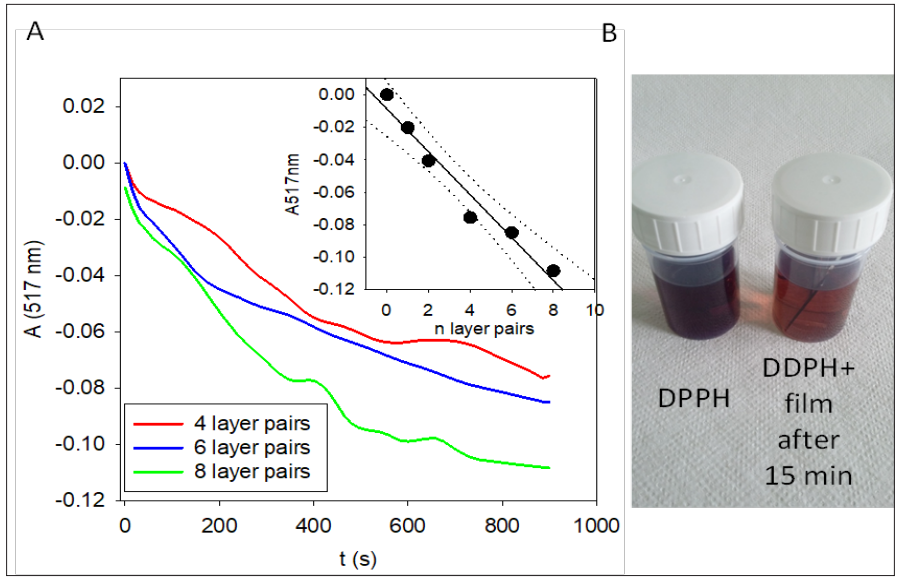

Figure 4: A: Antioxidant activity of (PAH-TA)n films as a function of the number of deposited layer pairs. The inset represents the reduction in absorbance at $\lambda=517 \mathrm{~nm}$ after $15 \mathrm{~min}$ of reaction between the quartz slide covered with a (PAH-TA)n film and the 10-4M DPPH solution. Full line and dashed lines represent the linear regression to the data and the limits of the $95 \%$ confidence interval respectively.

B: Picture of the DPPH solution (10-4M in absolute ethanol) and the DPPH solution containing a quartz slide with a (PAH-TA) 4 film on both its faces, after $15 \mathrm{~min}$ of reaction. 
The (PAH-TA $)_{n}$ films display some antioxidant activity as quantified through a decrease in absorbance at $\lambda=517 \mathrm{~nm}$, typical of the transformation of the purple DPPH into the yellowish DPPH-H (Figure 1). From a qualitative point of view, one observes indeed a reduction in the purple color of a $10^{-4} \mathrm{M}$ DPPH solution when a quartz slide covered with a (PAH-TA $)_{4}$ film on both its faces is immersed in it during $15 \mathrm{~min}$ (Figure 4A). After $15 \mathrm{~min}$ the reservoir of active TA in the films seems not yet exhausted as shown by the kinetic runs in Figure 4B. But for convenience we stopped the assays after $15 \mathrm{~min}$, and all films were compared for their antioxidant activity after this given reaction time with DPPH. It appears that the antioxidant activity of the (PAH-TA) films is proportional to the number of deposited layer pairs (Figure 4A) and hence to the amount of incorporated TA (Figure 2). This means that the films are permeable to DPPH which can reach all TA molecules in the film irrespective of their position, either close to the film/solution or to the quartz/film interface. This result is in perfect agreement with the data reported in ref. [11].

There is however a single way to suppress access of DPPH to the TA present in the (PAH-TA) films by capping them with (PAHPSS) layer pairs (Figure 5A\&5B). Such layer pairs are known to be of compact nature and to suppress the diffusion of a polyelectrolyte like poly-L-Lysine (PLL) from a superior (PLL-HA) ${ }_{\mathrm{m} 1}$ stratum (HA represents hyaluronic acid) to a previously deposited (PLL-HA) ${ }_{\mathrm{m} 2}$ stratum [14]. Hence (PAH-PSS) ${ }_{\mathrm{n}}$ layer pairs are able to stratify an otherwise un-stratified (exponentially growing) (PLL-HA) film. In the present investigation we show that an extremely thin (PAH-PSS) ${ }_{2}$ film (about 5-6nm in thickness [15]) is able to almost quantitatively suppress the diffusion of a small molecule like DPPH in the underlying (PAH-TA) $)_{3}$ film. This is the major finding of the present investigation. It could have some applications in packaging: when a (PAH-TA) ${ }_{n}$-(PAH-PSS) $)_{2}$ film is deposited on a package (for instance by alternated spray deposition to speed up the process with respect to alternated dipping) no color change of a drop of deposited DDPH is found as long as the coating has not undergone damage. But as soon as the package has been damaged, the (PAHPSS) 2 capping layer contains some cracks and becomes permeable to DPPH which then undergoes a visible and fast color change.

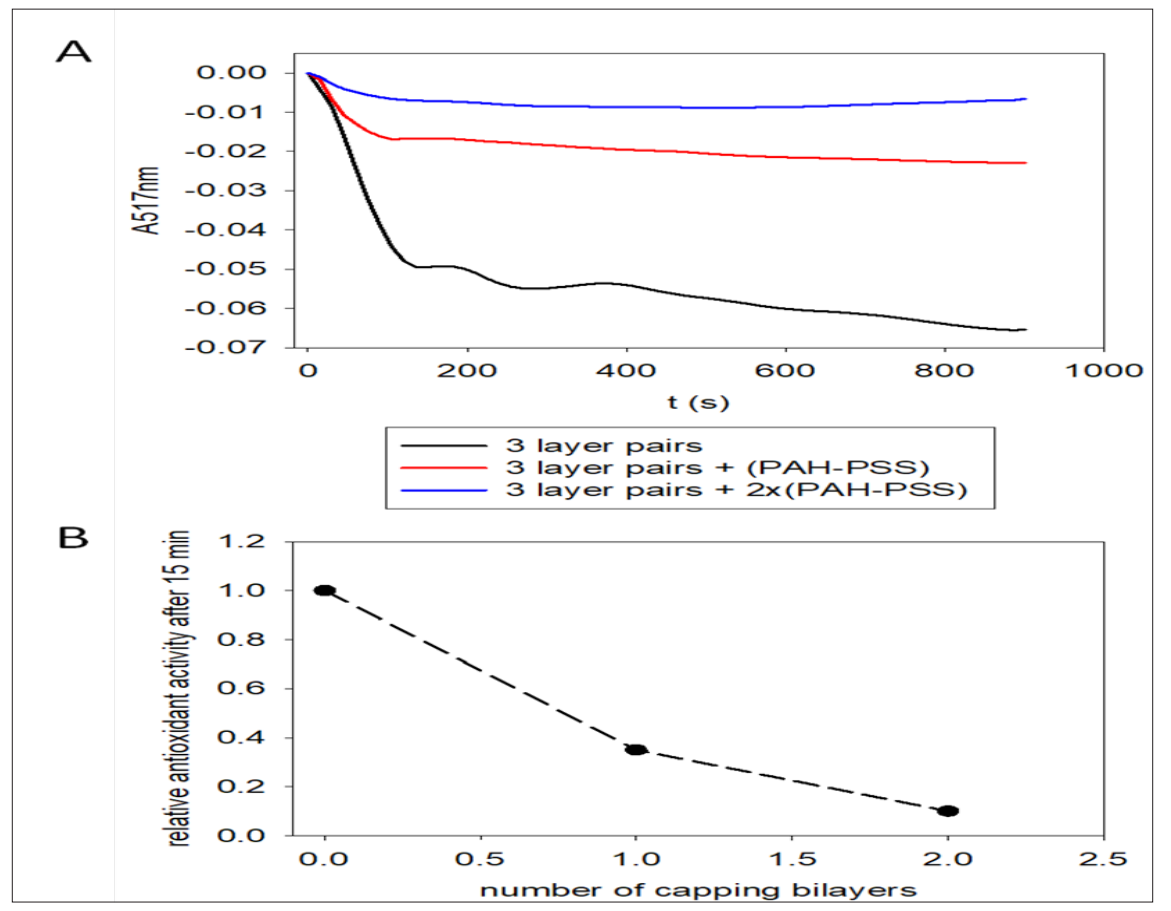

Figure 5: A: Reduction of the antioxidant activity of (PAH-TA)3 films after deposition of PAH-PSS "capping layers" as indicated in the inset. Each kinetic curve corresponds to an independently prepared film.

B: Evolution of the relative antioxidant activity after $15 \mathrm{~min}$ of contact with the $10-4 \mathrm{M}$ DPPH solution as a function of the number of (PAH-PSS) capping layer pairs. The relative activity of 1 corresponds to that of the (PAH-TA) film.

\section{Conclusion}

It has been shown that the antioxidant activity of (PAH-TA) films can be modulated through an increase in the number of deposited layer pairs and hence with an increase in the amount of TA, the antioxidant polyphenol, incorporated in the film. The addition of only two (PAH-PSS) capping layer pairs totally suppresses the access of DPPH to the underlying TA highlighting the compact and impermeable nature of those very thin capping layers. A practical application in possible sensing of packaging integrity is proposed on the basis of those findings.

\section{References}

1. Wang M, Simon JE, Aviles IF, He K, Zheng QY, et al. (2003) Analysis of antioxidative phenolic compounds in artichoke (Cynara scolymus L.). J Agric Food Chem 51(3): 601-608. 
2. Pokorný J (1991) Natural antioxidants for food use. Trends Food Sci \& Technol 2: 223-227.

3. Sileika TS, Barrett DG, Zhang R, Lau KHA, Messersmith PB (2013) Colorless multifunctional coatings inspired by polyphenols found in tea chocolate and wine. Angew Chem Int Ed 52: 10766-10770.

4. Barrett DG, Sileika TS, Messersmith PB (2014) Molecular diversity in phenolic and polyphenolic precursors of tannin-inspired nanocoatings. Chem Comm 50: 7265-7265.

5. Ejima H, Richardson JJ, Liang K, Best JP, Van Koeverden MP, et al. (2013) One-step assembly of coordination complexes for versatile film and particle engineering. Science 341(6142): 154-157.

6. Decher G (1997) Fuzzy nanoassemblies: Toward layered polymeric multicomposites. Science 277(5330): 1232-1237.

7. Erel Unal I, Sukhishvili SA (2008) Hydrogen-bonded multilayers of a neutral polymer and a polyphenol. Macromolecules 41(11): 3962-3970.

8. Shutava T, Prouty M, Kommireddy D, Lvov Y (2005) pH responsive decomposable layer-by-layer nanofilms and capsules on the basis of tannic acid. Macromolecules 38(7): 2850-2858.

9. Allais M, Meyer F, Ball V (2018) Multilayered films made from tannic acid and alkaline phosphatase with enzymatic activity and electrochemical behavior. J Colloid \& Interf Sci 512: 722-729.
10. Velmuguran P, Singam ERA, Jonnalagadda RR, Subramanian V (2013) Investigation on interaction of tannic acid with type i collagen and its effect on thermal, enzymatic, and conformational stability for tissue engineering applications. Biopolymers 101(5): 471-483.

11. Shutava TG, Prouty MD, Agabekov VE, Lvov YM (2006) Antioxidant properties of layer-by-layer films on the basis of tannic acid. Chem Lett 35(10): 1144-1145.

12. Ball V (2015) Stabilization of [poly(allylamine)-tannic acid] multilayer $_{\mathrm{n}}$ films in acidic and basic conditions after crosslinking with $\mathrm{NaIO}_{4}$. RSC Advances. 5: 55920-55925.

13. Yoo D, Shiratori SS, Rubner MF (1998) Controlling bilayer composition and surface wettability of sequentially adsorbed multilayers of weak polyelectrolytes. Macromolecules 31(13): 4309-4318.

14. Garza MJ, Schaaf P, Muller S, Ball V, Stoltz JF, et al. (2004) Multicompartment films made of alternate polyelectrolyte multilayers of exponential and linear growth. Langmuir 20(17): 7298-7302.

15. Ladam G, Schaad P, Voegel JC, Schaaf P, Decher G, et al. (2000) In situ determination of the structural properties of initially deposited polyelectrolyte multilayers. Langmuir 16(3): 1249-1255. 